\title{
OBSERVED TIMES OF THE MONTANA EARTHQUAKES, 1935*
}

\author{
By B. Gotenberg and C. F. Richter
}

UNUSUAL INTEREST attaches to seismograms of the earthquakes destructive at Helena, Montana, in October, 1935. The two principal shocks, at $4^{\mathrm{h}}$ on October 19 and $18^{\mathrm{h}}$ on October 31 (G.C.T.), were large enough for the $\mathrm{P}$ phases to be recorded at distances up to about $90^{\circ}$. The similarity of the seismograms, and the constancy of time differences between corresponding phases of the two shocks, show that they must have had nearly the same epicenter and focal depth. This epicenter must have been very close to the city of Helena; the considerable damage, and the great number of small associated shocks reported as felt and even as strong, are good noninstrumental evidence of this. In addition, part of the second large shock, and a large number of small shocks, were recorded at Helena by the United States Coast and Geodetic Survey. ${ }^{1}$ The $\mathrm{S}-\mathrm{P}$ interval on these records is about one or two seconds.

From the available data we have taken the epicenter at $46^{\circ} .6 \mathrm{~N}, 112^{\circ} .0 \mathrm{~W}$. (The U.S.C.\&G.S. publication gives $46^{\circ} 37^{\prime} \mathrm{N}, 111^{\circ} 58^{\prime}$ W.) The focal depth cannot have been more than a few kilometers. We have determined origin times from the $\mathrm{S}$ and $\mathrm{P}$ observations at distant stations as $04: 48: 03$ on October 19 and 18:37:49 on October 31. These data were taken from published station bulletins and from a few original seismograms.

Travel times for $\mathbf{P}$ and $\mathbf{S}$ are given in table 1, together with residuals against the travel-time tables given by the authors in their first and second papers on seismic waves. ${ }^{2}$

It will be noted that the residuals for $\mathbf{P}$ are generally small. Since these shocks are not large, the agreement is very satisfactory; there are not many late readings, such as might be expected from failure to record the first small motion. On the other hand, there are a few conspicuously early readings occurring in both shocks, as at St. Louis, Florissant, ${ }^{3}$ and Chicago. It is worth pointing out that in the Baffin Bay shock of November 20, 1933, residuals of -4 seconds at St. Louis and Florissant, and of -3 seconds at Little Rock, were found as a result of thorough study by A. W. Lee. ${ }^{4}$ In the Canadian shock of November 1, 1935, the reported arrivals of $\mathrm{P}$ at St. Louis, Florissant, and Chi-

* Manuscript received for publication January 17, 1938.

${ }^{1}$ Franklin P. Ulrich, "Helena Earthquakes," Bull. Seism. Soc. Am.,26:323-339 (1936); Frank Neumann, United States Earthquakes, 1935, U.S.C.\&G.S. Serial no. 600, pp. 42-56 and $67-80$ (1937).

${ }^{2}$ B. Gutenberg and C. F. Richter, "On Seismic Waves," Gerlands Beitr. z. Geophys., 43:56-133 (1934), 45: 280-360 (1935).

${ }^{3}$ We are indebted to the Rev. J. B. Macelwane, S. J., for the loan of original records at St. Louis, Florissant, and Little Rock for these shocks. We have verified the readings carefully, and there is no doubt about the early arrival of $P$.

4 "On the Travel of Seismic Waves from the Baffin Bay Earthquake of November 20, 1933," Geophysical Memoirs, no. 74 (London: Meteorological Office, 1937). 
TABLE 1

Travel Times and Residuals (min:sec) of P and S Waves in the Montana Earthouakes of October 19 (I) ANd October 31 (II), 1935

\begin{tabular}{|c|c|c|c|c|c|c|c|c|c|}
\hline \multirow{2}{*}{ Station } & \multirow{2}{*}{$\begin{array}{l}\text { Dis- } \\
\text { tance } \\
\text { in } \\
\text { deg. }\end{array}$} & \multicolumn{2}{|c|}{$\begin{array}{l}\text { Travel times of } \\
\text { P waves }\end{array}$} & \multicolumn{2}{|c|}{ Residuals } & \multicolumn{2}{|c|}{$\begin{array}{l}\text { Travel times of } \\
\text { S waves }\end{array}$} & \multicolumn{2}{|c|}{ Residuals } \\
\hline & & I & II & I & II & I & II & I & II \\
\hline 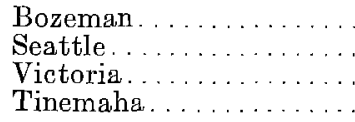 & $\begin{array}{r}1.1 \\
7.1 \\
7.9 \\
10.6\end{array}$ & $\begin{array}{l}2: 00 \\
2: 37\end{array}$ & $\begin{array}{l}0: 21 \\
1: 49\end{array}$ & $\begin{array}{l}2 \\
2\end{array}$ & $\begin{array}{r}-1 \\
2\end{array}$ & $\begin{array}{l}0: 41 \\
3: 12\end{array}$ & $\begin{array}{l}0: 43 \\
3: 13\end{array}$ & $\begin{array}{l}0 \\
0\end{array}$ & $\begin{array}{l}2 \\
1\end{array}$ \\
\hline 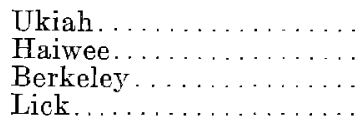 & $\begin{array}{l}11.1 \\
11.4 \\
11.6 \\
11.7\end{array}$ & $\begin{array}{l}2: 45 \\
2: 48 \\
2: 49 \\
2: 53\end{array}$ & $\begin{array}{l}2: 43 \\
2: 48 \\
2: 53\end{array}$ & $\begin{array}{l}3 \\
1 \\
0 \\
2\end{array}$ & $\begin{array}{l}1 \\
1 \\
4\end{array}$ & $\begin{array}{l}4: 46 \\
5: 09\end{array}$ & $5: 10$ & $\begin{array}{r}-4 \\
11\end{array}$ & -6 \\
\hline 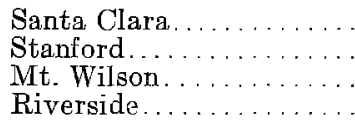 & $\begin{array}{l}11.8 \\
11.9 \\
13.2 \\
13.2\end{array}$ & $\begin{array}{l}2: 52 \\
3: 00 \\
3: 14 \\
3: 15\end{array}$ & $\begin{array}{l}3: 15 \\
3: 11\end{array}$ & $\begin{array}{l}0 \\
6 \\
2 \\
3\end{array}$ & $\begin{array}{r}3 \\
-1\end{array}$ & $\begin{array}{l}5: 14 \\
5: 26\end{array}$ & & $\begin{array}{r}6 \\
15\end{array}$ & \\
\hline 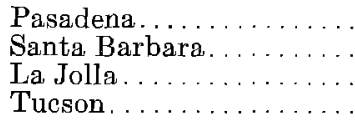 & $\begin{array}{l}13.3 \\
13.5 \\
14.3 \\
14.4\end{array}$ & $\begin{array}{l}3: 14 \\
3: 16 \\
3: 30 \\
3: 27\end{array}$ & $\begin{array}{l}3: 28 \\
3: 23\end{array}$ & $\begin{array}{r}1 \\
0 \\
3 \\
-1\end{array}$ & $\begin{array}{r}1 \\
-5\end{array}$ & 5: (45) & 5: $(59)$ & $(-1)$ & -1 \\
\hline 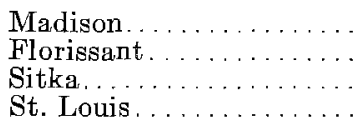 & $\begin{array}{l}16.4 \\
17.6 \\
17.7 \\
17.8\end{array}$ & $\begin{array}{l}4: 02 \\
4: 07 \\
4: 04\end{array}$ & $\begin{array}{l}3: 54 \\
4: 03 \\
4: 12\end{array}$ & $\begin{array}{l}-6 \\
-2 \\
-7\end{array}$ & $\begin{array}{r}0 \\
-5 \\
3\end{array}$ & $\begin{array}{l}7: 21 \\
7: 36 \\
7: 33\end{array}$ & $\begin{array}{l}7: 01 \\
7: 22 \\
7: 38\end{array}$ & $\begin{array}{r}-6 \\
7 \\
2\end{array}$ & $\begin{array}{r}1 \\
-5 \\
9\end{array}$ \\
\hline 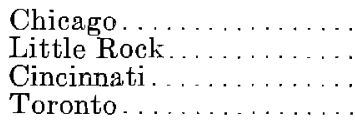 & $\begin{array}{l}18.1 \\
19.0 \\
21.4 \\
23.1\end{array}$ & $\begin{array}{l}4: 08 \\
4: 20 \\
4: 49 \\
5: 10\end{array}$ & $\begin{array}{l}4: 07 \\
4: 26 \\
5: 08\end{array}$ & $\begin{array}{r}-6 \\
-4 \\
-1 \\
2\end{array}$ & $\begin{array}{r}-7 \\
2\end{array}$ & $\begin{array}{l}7: 29 \\
7: 50 \\
8: 53 \\
9: 18\end{array}$ & $\begin{array}{l}7: 31 \\
8: 03 \\
9: 18\end{array}$ & $\begin{array}{r}-8 \\
-5 \\
10 \\
4\end{array}$ & $\begin{array}{r}-6 \\
8\end{array}$ \\
\hline 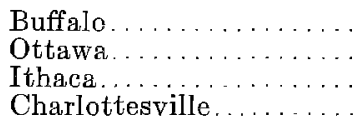 & $\begin{array}{l}23.7 \\
25.1 \\
25.5 \\
26.0\end{array}$ & $\begin{array}{l}5: 14 \\
5: 25 \\
5: 33\end{array}$ & $\begin{array}{l}5: 15 \\
5: 29 \\
5: 35\end{array}$ & $\begin{array}{r}0 \\
-3 \\
1\end{array}$ & $\begin{array}{l}1 \\
1 \\
3\end{array}$ & $\begin{array}{r}9: 37 \\
9: 45 \\
10: 13 \\
10: 08\end{array}$ & $\begin{array}{r}9: 35 \\
9: 51 \\
10: 17 \\
10: 10\end{array}$ & $\begin{array}{r}13 \\
-6 \\
15 \\
1\end{array}$ & $\begin{array}{r}11 \\
0 \\
19 \\
3\end{array}$ \\
\hline 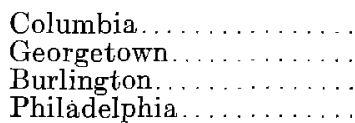 & $\begin{array}{l}26.6 \\
26.6 \\
27.1 \\
27.4\end{array}$ & $5: 40$ & $5: 41$ & -2 & 1 & $\begin{array}{l}10: 07 \\
10: 11 \\
10: 25 \\
10: 27\end{array}$ & $\begin{array}{l}10: 08 \\
10: 21 \\
10: 27\end{array}$ & $\begin{array}{r}-10 \\
-6 \\
0 \\
-6\end{array}$ & $\begin{array}{l}-9 \\
-4 \\
-6\end{array}$ \\
\hline $\begin{array}{l}\text { Fordham } \ldots \ldots \ldots \ldots \\
\text { Harvard } \ldots \ldots \ldots \ldots \ldots \\
\text { Huancayo } \ldots \ldots \ldots \ldots \ldots \\
\text { Stuttgart } \ldots \ldots \ldots \ldots \ldots\end{array}$ & $\begin{array}{l}27.9 \\
28.9 \\
67.0 \\
72.1\end{array}$ & $\begin{array}{r}5: 56 \\
5: 59 \\
10: 55 \\
11: 27\end{array}$ & $\begin{array}{r}6: 00 \\
10: 54 \\
11: 28\end{array}$ & $\begin{array}{r}3 \\
-2 \\
-2 \\
0\end{array}$ & $\begin{array}{r}-1 \\
-3 \\
1\end{array}$ & $\begin{array}{l}10: 32 \\
10: 55 \\
19: 39\end{array}$ & $10: 56$ & $\begin{array}{r}-6 \\
2 \\
-5\end{array}$ & 3 \\
\hline 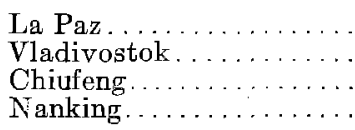 & $\begin{array}{l}74.2 \\
74.3 \\
83.6 \\
89.3\end{array}$ & $11: 47$ & $11: 41$ & 8 & 2 & & $\begin{array}{l}21: 15 \\
22: 55 \\
23: 47\end{array}$ & & $\begin{array}{r}11 \\
10 \\
0\end{array}$ \\
\hline $\begin{array}{l}\text { Tiflis. . . . . . . . . . . . . . . . . . . . } \\
\text { Tashkent . . }\end{array}$ & $\begin{array}{l}89.7 \\
92.5\end{array}$ & & $\begin{array}{l}13: 03 \\
13: 15\end{array}$ & & $\begin{array}{l}2 \\
2\end{array}$ & & $23: 41$ & & -10 \\
\hline
\end{tabular}


cago are about 4 seconds early relative to the times reported by other stations. There are no such early arrivals for the Southern California earthquakes of March 11, 1933, and March 25, 1937; in the Cuban shock of February 3, 1932, any effect of this kind is within the limits of error. The appearance is that of a velocity above average for $\mathrm{P}$ waves arriving at the stations in question from northerly directions; but more data of strong shocks at the shorter distances will be needed to establish the reality of this hypothetical effect.

As is usual at short distances, the residuals for the $\mathbf{S}$ readings show a considerable scatter, and no valuable conclusions can be drawn from the reported data. Other phases are very scantily represented.

[Balch Graduate School of the Geological Sclences, California Institute of Technology, Pasadena, CONTRIBUTION No. 248] 